\title{
Longitudinal phase space synthesis with tailored 3D-printable dielectric-lined waveguides
}

\author{
F. Mayet๑, ${ }^{*}$ R. Assmann, and F. Lemery ${ }^{\dagger}$ \\ DESY, Notkestrasse 85, 22607 Hamburg, Germany
}

(Received 28 September 2020; accepted 23 November 2020; published 14 December 2020)

\begin{abstract}
Longitudinal phase space (LPS) manipulation is critical and necessary for advanced acceleration concepts, radiation sources and improving the performance of x-ray free electron lasers. Here we present a simple and versatile method to semiarbitrarily shape the longitudinal phase space of a charged bunch by using wakefields generated in tailored dielectric-lined waveguides. We apply the concept in simulation and provide examples for radiation generation and bunch compression. We finally discuss the manufacturing capabilities of a modern 3D printer and investigate how printing limitations, as well as the shape of the input LPS, affect the performance of the device.
\end{abstract}

DOI: $10.1103 /$ PhysRevAccelBeams.23.121302

\section{INTRODUCTION}

Emerging advanced accelerator concepts require precise control over the longitudinal phase space (LPS) of charged particle beams. Efficient beam-driven acceleration, for example, relies on longitudinally tailored electron bunch profiles which may be produced with an appropriate energy modulation and dispersive section [1-4] or other means [5-8]. Phase-space linearization for bunch compression is especially important to optimize the performance of multistage linacs and x-ray free electron lasers (XFELs) [9-11]. There are several ways to control the LPS. Energy modulation approaches via self-wakes in e.g., dielectric or corrugated structures provide attractive and simple methods to produce microbunch trains and large peak currents $[3,12,13]$. Laser-based energy modulation techniques using magnetic chicanes are particularly useful for FEL seeding [14-18] and for beam acceleration [19]. Arbitrary laser-based phase space control was discussed in [20], illustrating the potential for producing different current profiles for various applications. Unfortunately however, although the scheme works well in simulation, the approach is complex to implement, requiring several undulators and magnetic chicanes in addition to the modulating laser.

In this paper we explore arbitrary waveform synthesis using self-wakes produced in dielectric-lined waveguides

\footnotetext{
*frank.mayet@desy.de

†rancois.lemery@desy.de
}

Published by the American Physical Society under the terms of the Creative Commons Attribution 4.0 International license. Further distribution of this work must maintain attribution to the author(s) and the published article's title, journal citation, and DOI.
(DLWs). By using segmented waveguides with varying cross sections, the excited wakefields carry different spectral contents throughout the structure, enabling control over the energy modulation across the bunch. The dependence of the modal content on the DLW geometry allows for enough degrees of freedom to optimize such a segmented structure according to the desired output LPS. Due to the nature of the physical process, the scheme is completely passive, removing the need for synchronization with e.g., a modulating laser beam or rf field. This is, for example, also taken advantage of in [21], where the authors use a leading drive bunch to excite the wakefields. In the following, the device is referred to as a longitudinal phase space shaper (LPSS).

The paper is structured as follows: Section II provides an overview on 1D wakefield theory, Sec. III discusses Fourier synthesis for single-mode structures, Sec. IV provides examples for multimode structure optimizations using computational optimization, Sec. V discusses the transition from idealized to realistically shaped structures. We also study the impact of limited printing resolution of modern 3D printers, which we propose to be used to manufacture the LPSS devices. Finally, Sec. VI discusses the effect of slight variations in the shape of the input LPS, as well as systematic manufacturing errors on a figure of merit of an output LPS, based on an example optimization case.

\section{WAKEFIELD GENERATION IN A DLW}

The theory of Cherenkov wakefield generation in cylindrically symmetric DLWs is well described in [22-24]. Here we follow [23], for a structure with inner radius $r=a$, outer radius $r=b$ and dielectric permittivity $\epsilon_{r}$. The outer surface is assumed to be coated with a perfect conductor. See Fig. 1 for a schematic. A more rigorous theoretical 


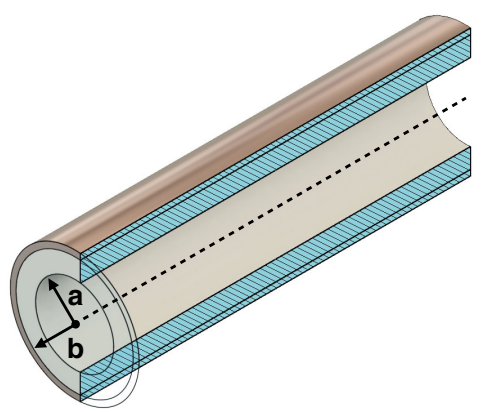

FIG. 1. Schematic of a cylindrical dielectric-lined waveguide. The lining with dielectric constant $\epsilon_{r}$ has an inner radius $a$ and an outer radius $b$. It is coated with a thin metallic layer on the outside.

investigation could include conductive and dielectric losses in DLWs $[25,26]$.

In the ultrarelativistic limit, a point charge traveling on axis $(r=0)$ will excite a wakefield with a corresponding Green's function with $M$ modes [27,28],

$$
G(t)=\sum_{m=1}^{M} \kappa_{m} \cdot \cos \left(2 \pi f_{m} t\right),
$$

where $\kappa_{m}$ and $f_{m}$ are the loss factor and frequency of the $m$ th mode respectively and are calculated numerically $[23,29]$. This Green's function is often also referred to as the single particle wake potential $W_{z}(\tau)[V / C]$, where $\tau$ denotes the time difference between the point charge and a trailing witness charge. Note that it is defined by the boundary conditions and hence-in our case- the geometry of the DLW. By varying e.g., the inner radius $a$ of a DLW, it can be seen that both wavelength and amplitude depend strongly on the geometry of the structure (see Fig. 2). Considering that the amplitude of the longitudinal wakefield scales as $1 / a^{2}$ [23], it becomes apparent that potentially very high field strengths can be reached in small aperture DLWs.
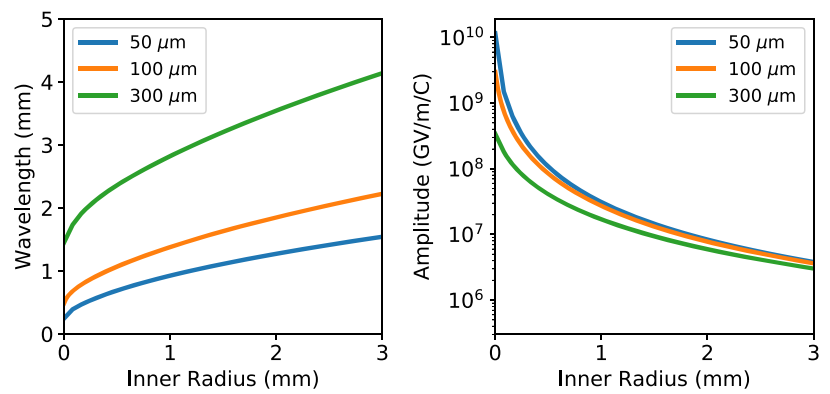

FIG. 2. Plot of the numerically calculated wavelength and amplitude of a wakefield excited by an on-axis electron bunch in a single-mode DLW. The different colors correspond to different thicknesses of the dielectric lining.
The overall wake potential $V(t)$ produced by a bunch can be calculated by convolving its current profile $I(t)$ with $W_{z}(\tau)$. Therefore

$$
V(t)=-\int_{-\infty}^{t} I(\tau) W_{z}(t-\tau) d \tau
$$

The field excitation can also be described in terms of the frequency dependent bunch form factor $F_{m}$. Then

$$
V(t)=q \cdot \sum_{m=1}^{M} F_{m} \kappa_{m} \cdot \cos \left(2 \pi f_{m} t\right)
$$

where $q$ is the total charge of the bunch. A strong mode excitation therefore requires a bunch with an appropriate spectral content i.e., a relatively short bunch, or also by having a relatively short rise time in e.g., a flattop distribution $[13,30]$.

A cascaded arrangement of multiple DLWs allows the energy modulations via wakefields from the different structures to be concatenated. The following section illustrates the broad potential for a set of cascaded, or a single segmented structure to produce a versatile range of energy modulations. We note that the usage of segmented structures, and the produced effects of transient wakes is discussed in Sec. V.

\section{LPS SHAPING IN SINGLE-MODE STRUCTURES}

Fourier synthesis provides a simple way to produce a large variety of waveforms which have various applications in conventional electronics. Here we explore how Fourier synthesis can be applied to charged particle beams using self-wakes imparted in high-impedance mediums, e.g., DLWs. We are specifically interested in the Fourier series for odd functions, since the wakefield at the head of the bunch is zero.

In the simplest case, each of the individual segments of an LPSS is a single-mode structure with a specific fundamental mode frequency and amplitude. As discussed above, the wake function $W_{z}(\tau)$ for such a structure is simply given by

$$
W_{z, m}(\tau)=\kappa_{m} \cdot \cos \left(2 \pi f_{m} \tau\right)
$$

Using this and Eq. (2), the energy modulation imparted by a single DLW segment $n$ can be estimated as (cf. [31])

$$
\begin{aligned}
\Delta E_{n}(t)= & -l_{n} \cdot \kappa_{m(n)} \\
& \cdot \int_{-\infty}^{t} I_{n-1}(\tau) \cos \left[2 \pi f_{m(n)}(t-\tau)\right] d \tau,
\end{aligned}
$$

where $l_{n}$ is the length of the $n$th DLW segment. The $n-1$ subscript in $I_{n-1}(\tau)$ refers to the fact that the current profile 
might change after each segment, if longitudinal dispersion is significant (i.e., in low energy regimes). For simplicity we assume an instantaneous change in $I(\tau)$ after each segment, which in reality will, of course, happen gradually. We note that for sufficiently high energies, this effect is negligible and can in principle also be taken into account. The total energy modulation imparted by an $N$-segment structure can hence be estimated as

$$
\Delta E_{\mathrm{tot}}(t)=\sum_{n=0}^{N} \Delta E_{n}(t)
$$

(see Sec. V for a discussion on the effects of sharp segment transitions on the resulting wakefields). Here it is assumed that the current profile does not change along the structure, i.e., the longitudinal dispersion is very small. Assuming a flattop current profile with hard edges $I(\tau)$, the total energy modulation reduces to

$$
\Delta E_{\mathrm{tot}}(t)=\sum_{n=0}^{N} A_{m(n)} \cdot \sin \left(2 \pi f_{m(n)} t\right),
$$

where $A_{m(n)} \propto l_{n} \kappa_{m(n)}$ is the amplitude factor of the $n$th segment. Considering the scaling laws shown in Fig. 2, arbitrary LPS shapes can be constructed via Fourier composition. The amplitude $A_{m(n)}$ of each frequency component can be adjusted by choosing an appropriate $l_{n}$. It should be noted that the frequency content of the input current profile must be sufficient to excite the desired modes.

Equation (7) essentially corresponds to an ordinary Fourier sine series. A sawtooth wave, for example, can be constructed by summing up only even harmonics with proper normalization. Hence, the Fourier series for a given fundamental frequency $f_{0}$ is given by

$$
F_{\text {saw }}(t)=A \cdot \sum_{n=0}^{\infty} \frac{1}{2 n+2} \sin \left[\pi(2 n+2) f_{0} \cdot t\right],
$$

where $A$ is an amplitude scaling factor. Another simple example is a square wave. Its Fourier series only contains odd harmonics. Thus

$$
F_{\text {squ }}(t)=A \cdot \sum_{n=0}^{\infty} \frac{1}{2 n+1} \sin \left[2 \pi(2 n+1) f_{0} \cdot t\right]
$$

Figure 3 visualizes the two modulation types for different values of $N$.

In order to explore possible use cases of such energy modulations we investigated the effect of applying linear longitudinal dispersion $\left(R_{56}\right)$ to the phase space. In this work we adopt the convention that the head of the bunch is at $z<0$ and hence $R_{56}<0$ in a drift, or typical four-dipole chicane. Figure 4 shows contour plots of both the beam current within a single fundamental modulation wavelength
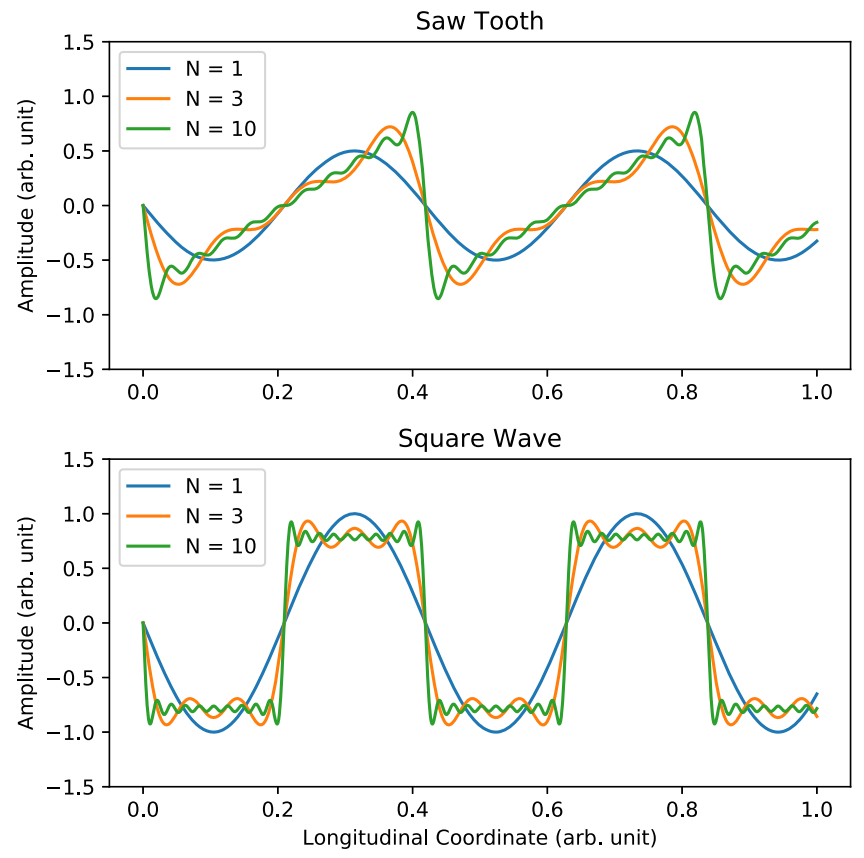

FIG. 3. Plot of amplitude vs longitudinal coordinate for an arbitrary sawtooth modulation [Eq. (8)] and an arbitrary square wave modulation [Eq. (9)] for $N=1, N=3$ and $N=10$.

$\lambda_{0}=1 \mathrm{~mm}$, as well as the frequency content of the bunch vs the longitudinal dispersion $R_{56}$ for different values of $N$. The idealized input current is assumed to be flattop. We also assume a cold beam in order to be able to explore the full mathematical limits of the scheme. The investigation is carried out for both a sawtooth modulation [cf. Eq. (8)], as well as for a square wave modulation [cf. Eq. (9)]. It can be seen, as longitudinal dispersion is applied, interesting features emerge.

In the case of the sawtooth modulation, first the modulation on the slowly rising part of the sawtooth (see Fig. 3) is compressed. Then, as $\left|R_{56}\right|$ increases, the minimum and maximum of the sawtooth converge, which results in a current spike. The current spike is more defined as $N$ increases, which can be attributed to a less pronounced Gibbs ringing at the sharp edges of the sawtooth, as well as an overall flattening for higher values of $N$. Gibbs ringing, in general, refers to an overshoot of the Fourier series at sharp discontinuities, which is more significant the lower the number of terms in the Fourier series. This behavior is also represented by the ellipsoidal shape visible in the contour plots of the beam current vs $\mathrm{d} z$ and $R_{56}$, which becomes narrower as $N$ increases (see Fig. 4). It is interesting to note that as the amplitude of the high frequency modulation along the rising part of the sawtooth varies, different parts of the rising edge require different values of $R_{56}$ for optimal compression. This is clearly visible in the contour plots. For symmetry reasons, always two submicrobunches emerge. By adjusting $R_{56}$, a specific pair of microbunches with a defined relative distance can be 
Saw Tooth Modulation
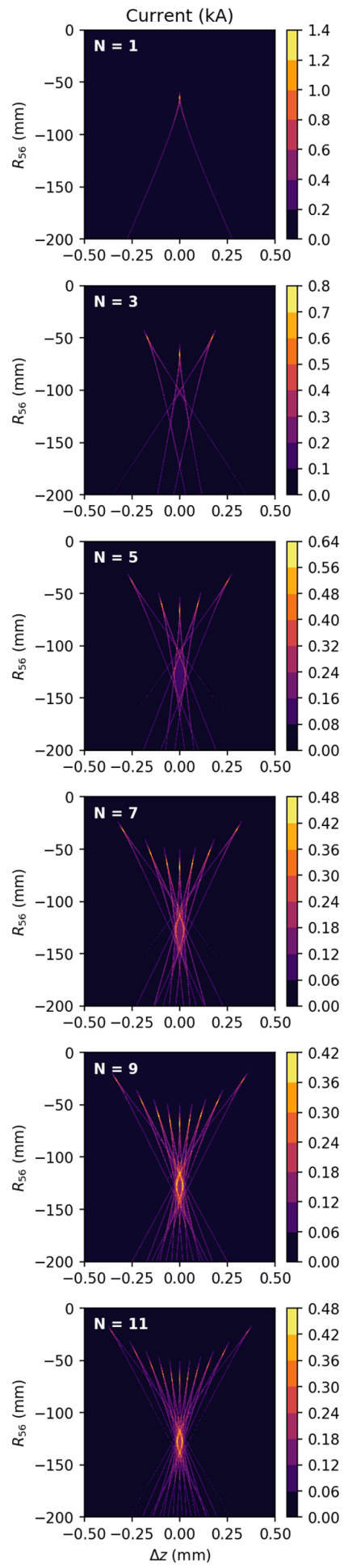
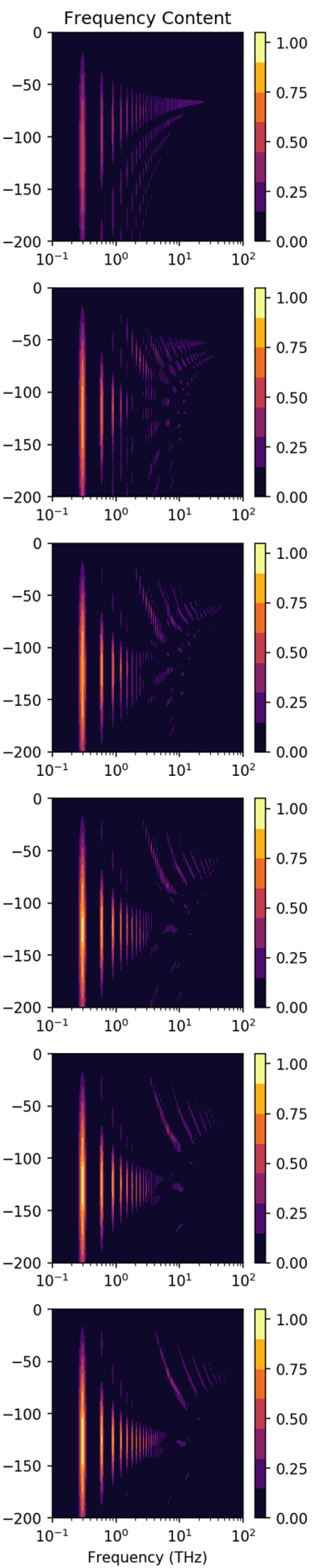

Square Wave Modulation
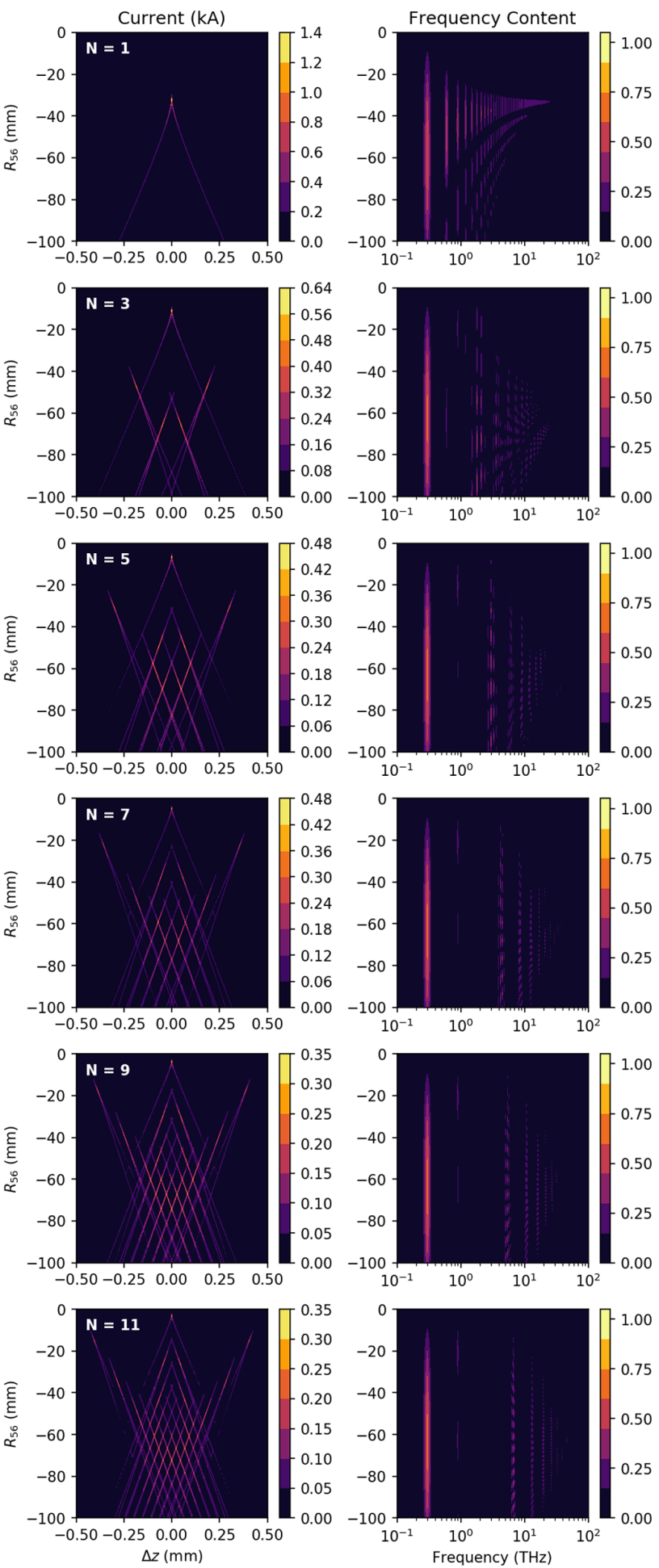

FIG. 4. Contour plots of both the beam current within a single fundamental modulation wavelength $\lambda_{0}=1 \mathrm{~mm}$, as well as the frequency content of the bunch vs the longitudinal dispersion $R_{56}$. Here, $\Delta z=z-\lambda_{0} / 2$. The scan was performed for $N \in[1,3,5,7,9,11]$. Both a sawtooth modulation according to Eq. (8), as well as a square wave modulation according to Eq. (9) are shown. The idealized input current is assumed to be of flattop shape and the initial energy $E_{0}=100 \mathrm{MeV}$ is constant along the bunch. It has a total bunch length of $1 \mathrm{~mm}$ and $Q=42 \mathrm{pC}$. The assumed maximum modulation depth of the lowest frequency component is $500 \mathrm{keV}$. Note that a high slice energy spread would lead to blurring out the small features in the respective phase spaces. Here we assume a cold beam in order to explore the full mathematical potential of the scheme. 
selected. It has to be noted, however, that-depending on the modulation depth-these substructures require very low slice energy spread to be significant vs the background. If the respective bunching factor $b_{n}$ should not be reduced by more than roughly a factor of 2 , then $\delta_{\text {mod }} / \delta_{\mathrm{sl}} \leq n$ has to be satisfied, where $n$ is the harmonic number of $f_{0}$ and $\delta_{\text {mod }}$ and $\delta_{\mathrm{sl}}$ are the relative modulation depth and slice energy spread respectively; cf. [32].

In the case of the square wave modulation the plots show a different behavior. As $\left|R_{56}\right|$ increases, first a single current spike is formed, which corresponds to the sharp edge of the energy modulation. As the edge becomes sharper (higher $N$ ), optimal bunching occurs for weaker $R_{56}$. Increasing $\left|R_{56}\right|$ beyond optimal bunching reveals a very particular rhombus pattern in the contour plot, which is explained by the fact that the minimum and maximum parts of the square wave are shifted on top of each other (the higher energy part towards smaller $z$ and the lower energy part towards larger $z$ ). The higher the value of $N$, the more intricate the rhombus pattern becomes. It is interesting to note that - by applying appropriately strong $R_{56}$ - the modulation on the (ideally) constant parts of the square wave will form two submicrobunch trains at their own distinct energy levels ( $E_{0} \pm \Delta E$, where $\Delta E$ refers to the amplitude of the ideal square wave).

The sawtooth and square wave modulation are only two examples of possible Fourier series based LPS modulations. Many other interesting waveforms might exist, which are not discussed here. In order to show how drastic even
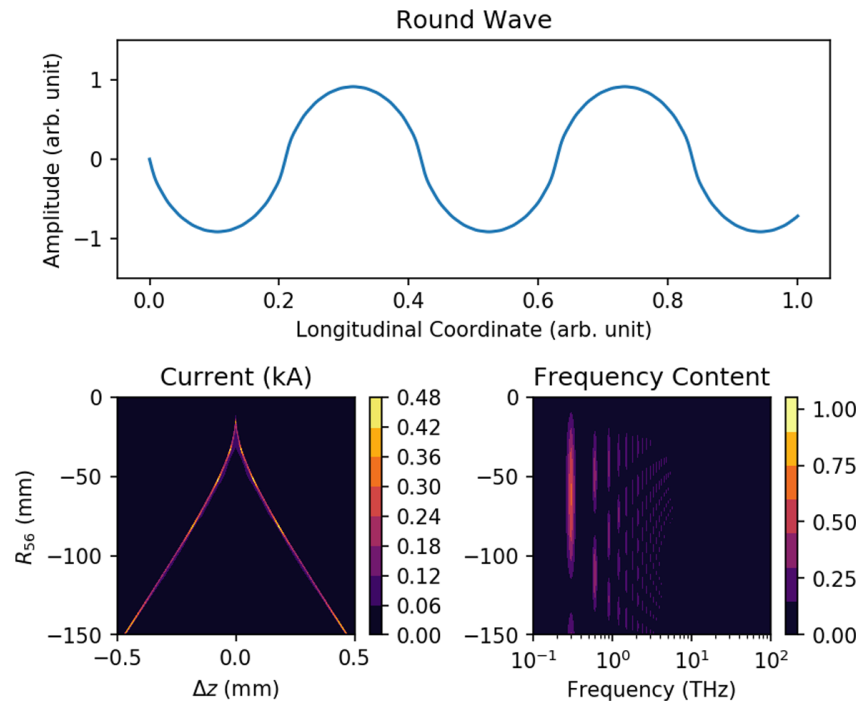

FIG. 5. Top: plot of an $N=11$ round wave modulation according to Eq. (10). Bottom: contour plots of both the beam current within a single fundamental modulation wavelength $\lambda_{0}=1 \mathrm{~mm}$, as well as the frequency content of the bunch vs the longitudinal dispersion $R_{56}$. Here, $\Delta z=z-\lambda_{0} / 2$. The scan was performed for $N=11$. The idealized input current and modulation depth is assumed to be the same as described in Fig. 4. small changes to a particular Fourier series definition can be, one can consider squaring the normalization factor in Eq. (9). This yields, instead of a sharp square wave, a smooth rounded wave. The definition now reads

$$
F_{\text {rnd }}(t)=A \cdot \sum_{n=0}^{\infty} \frac{1}{(2 n+1)^{2}} \sin \left[2 \pi(2 n+1) f_{0} \cdot t\right] .
$$

Figure 5 shows both the shape of an $N=11$ round wave modulation, as well as contour plots analogous to Fig. 4. It can be seen that applying $R_{56}$ to this kind of modulation at first glance leads to a dependence similar to a simple sine wave modulation. The main difference, however, is that the beam current of the submicrobunches, which occur after overbunching, shows multiple additional maxima of similar magnitude compared to the initial single microbunch. In the case of a simple sine modulation the peak current would decrease rapidly. As the number of additional maxima increases with $N$, this means that using a high- $N$ round wave modulation, one can obtain high-quality submicrobunches with semicontinuously adjustable relative spacing.

\section{ARBITRARY MULTIMODE OPTIMIZATION}

So far we have investigated Fourier shaping of an idealized input LPS. In order to work with arbitrary input distributions, a more sophisticated optimization routine must be used. This is especially true if multimode DLW segments are to be included, as the number of degrees of freedom gets too large for manual optimization. Hence a routine based for example on the particle swarm algorithm (PSO) must be employed [33]. The algorithm varies all geometric parameters of the individual segments at the same time in order to find a global minimum of a given merit function. This merit function is given in the LPSS case by the similarity of the resulting LPS to the desired LPS shape. Since segment radius, length and wall thickness can be varied, the resulting number of independent variables is $3 N$, where $N$ is the number of segments. For the LPSS study presented here, the PSO was implemented using PyOpt [34]. At each iteration step either a simulation using a specifically generated input file for a numerical simulation code, or a semianalytical calculation based on Eq. (2) is carried out. If space-charge effects are neglected, the difference between the numerical simulation using ASTRA [35] and the semianalytical approach was found to be negligible. Hence, the much faster semianalytical calculation was used for the simulations shown in the following discussion.

As an example optimization goal the linearization of an incoming LPS obtained from close to on-crest acceleration was chosen. This scenario is interesting, because the resulting LPS shows a clear signature of the sinusoidal rf field of the linac structures, which would limit the achievable bunch length in subsequent compression. 
TABLE I. LPSS optimization variable ranges for each of the ten segments.

\begin{tabular}{lc}
\hline \hline Parameter & Value \\
\hline Inner radius & {$[0.1,2.5] \mathrm{mm}$} \\
Dielectric thickness & {$[50,1000] \mu \mathrm{m}$} \\
Segment length & {$[1,100] \mathrm{mm}$} \\
\hline \hline
\end{tabular}

In order to keep the number of free parameters manageable, the number of LPSS segments was limited to 10. The optimizer was configured to bring the Pearson's $R$ value of centered $\tilde{n} \sigma_{z}$ regions within the final distribution as close to 1 as possible. Here $\tilde{n} \in \mathbb{N}$ and $R$ is defined as

$$
R=\frac{\sum(x-\bar{x})(y-\bar{y})}{\sqrt{\sum(x-\bar{x})^{2} \cdot \sum(y-\bar{y})^{2}}} \in[-1,1],
$$

where $\bar{x}$ and $\bar{y}$ denote the mean of $x$ and $y$ respectively. $R$ is a measure of the degree of linear correlation between $x$ and $y$. In our case $x$ is the longitudinal coordinate and $y$ the energy. Table I summarizes the possible ranges of values for the geometry parameters of the ten individual segments.

For the input we consider three different electron bunch distributions with 10,100 and $200 \mathrm{pC}$ of total charge and a mean energy of $\sim 100 \mathrm{MeV}$, based on numerical simulations of the ARES linac at DESY [36]. This is done in order to provide a realistic example, which could be used as the basis for future experimental verification of the scheme. Figure 6 shows a schematic of the ARES lattice. If both linac structures are driven at their respective maximum gradients of $\sim 25 \mathrm{MV} / \mathrm{m}$, a final mean energy up to $\sim 150 \mathrm{MeV}$ is possible. The decision to limit the example working points to $\sim 100 \mathrm{MeV}$ is a practical one, as the overall LPSS structure length generally increases with the required LPS modulation strength and the experimental chamber at ARES imposes strict space limitations. The three working points were optimized to minimize transverse emittance at the interaction point $(z=16.8 \mathrm{~m})$ for three different charges using ASTRA, including space charge effects. Table II summarizes the respective beam parameters.

In order to first investigate the effect of limiting the optimization goal to specific $\tilde{n} \sigma_{z}$ regions within the LPS on the resulting LPSS geometry, four different optimization
TABLE II. Beam parameters of the three ARES linac working points (WP) at the interaction point $(z=16.8 \mathrm{~m})$, obtained from ASTRA simulations. Initial spatial and temporal profile: Gaussian. TWS: traveling wave structure.

\begin{tabular}{lccc}
\hline \hline Parameter & WP1 & WP2 & WP3 \\
\hline Charge & $10 \mathrm{pC}$ & $100 \mathrm{pC}$ & $200 \mathrm{pC}$ \\
TWS injection phase & $-3^{\circ}$ & $-5^{\circ}$ & $-8^{\circ}$ \\
$E_{0}$ & $108 \mathrm{MeV}$ & $109 \mathrm{MeV}$ & $108 \mathrm{MeV}$ \\
$\sigma_{E} / E_{0}$ & $2.8 \times 10^{-4}$ & $4.1 \times 10^{-3}$ & $5.3 \times 10^{-3}$ \\
$\sigma_{t}$ & $673 \mathrm{fs}$ & $1.95 \mathrm{ps}$ & $2.65 \mathrm{ps}$ \\
$\varepsilon_{\mathrm{n}, x, y}$ & $146 \mathrm{~nm}$ & $370 \mathrm{~nm}$ & $465 \mathrm{~nm}$ \\
\hline \hline
\end{tabular}

runs were performed. As input, WP3 was chosen (see Table II). For now the LPSS structures are simulated as ten cascaded individual DLWs (see Sec. V for a discussion on the implications of this approach). Figure 7 shows the results. Starting from an overall linearity of the input LPS of $R=0.9568$, it can be seen that in all cases the use of the LPSS improved the linearity significantly. The smaller the region of interest (ROI) within the LPS, the better the results, reaching up to $R=0.999998$ in the case of $\tilde{n}=1$. It is apparent that if the whole LPS is taken into account (i.e., a $6 \sigma$ ROI), the results are noticeably worse than for a restricted ROI. This can be attributed to the fact that, due to the Gaussian time profile of the input LPS, the beam current in the head region of the bunch is low and hence the strength of the excited wakefield is weak. Thus, it is difficult for the optimizer to find configurations where this region is linearized sufficiently well, subsequently spoiling the overall linearity of the LPS. Excluding this head region of the LPS from the optimization, on the other hand, improves the performance significantly. In addition, smaller ROIs contain less strong LPS curvature in this particular example. In an experiment at ARES for example, the region outside of the ROI could be cut using the slit collimator implemented in the magnetic compressor (see below).

In addition to the degree of linearity in the respective $\tilde{n} \sigma_{z}$ region, Fig. 7 also shows that two important geometry parameters depend on the ROI as well. First, the overall structure length decreases with the ROI. This is of practical importance, not only in terms of beam transport through the structure, but also in terms of manufacturing. Second, the

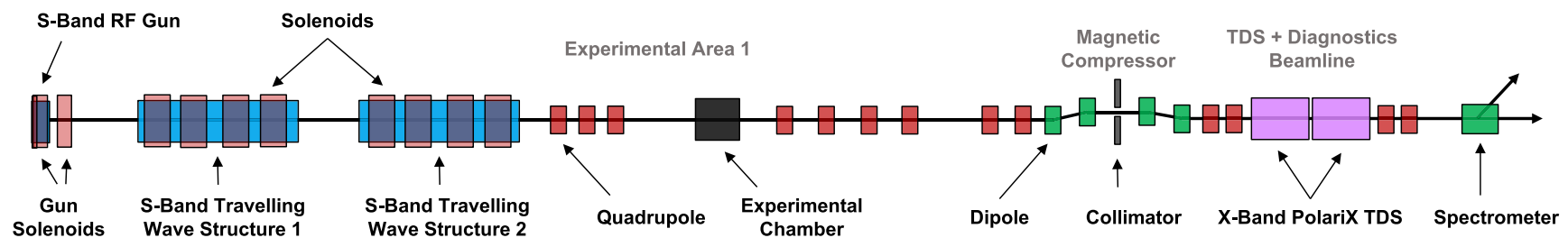

FIG. 6. Schematic of the layout of the ARES linac at DESY (not to scale). The LPSS interaction is simulated to take place in the experimental chamber of Experimental Area 1 at $z=16.8 \mathrm{~m}$. 

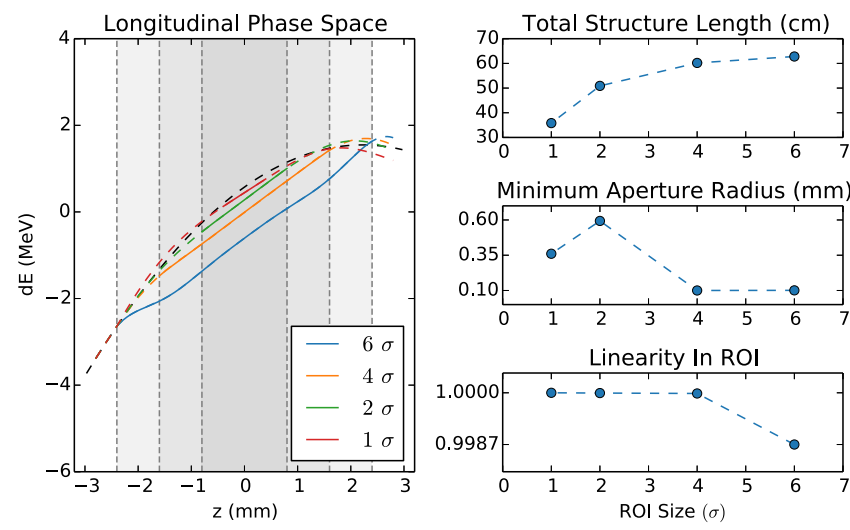

FIG. 7. Left: comparison of LPSS linearization results, depending on the size of a defined region of interest (ROI) within the bunch. The solid part of the lines corresponds to the respective ROI. The black curve corresponds to the initial LPS. Right: total LPSS structure length, minimal segment aperture radius within the structure and linearity of the output LPS within the respective ROI, depending on the ROI size. minimum aperture radius of the structure increases with a decreasing ROI, which is important from a beam transport point of view and in accordance with the current profile of the bunch and the dependence of the wakefield strength vs the aperture radius $\left(E_{z} \propto 1 / a^{2}\right.$; see Fig. 2). If the head of the bunch is included in the ROI, the optimizer will have to use small apertures in order to compensate for the low current in the head region of the bunch. Taking these results into account, it is clearly worth considering trading-in case of a Gaussian time profile-less than $5 \%$ of the total bunch charge for the much better linearization performance of a $4 \sigma$ ROI.

Based on the results discussed above, optimization runs were performed for all of the three ARES working points, considering both a full $6 \sigma$ and a smaller $2 \sigma$ linearization ROI. Note that from WP1 to WP3 both charge and bunch length increases. Figure 8 shows the detailed results. It can be seen that in all cases a significant improvement of $R$ can be achieved within the ROI. Better results are obtained in the case of the limited ROI, as expected. Furthermore, the
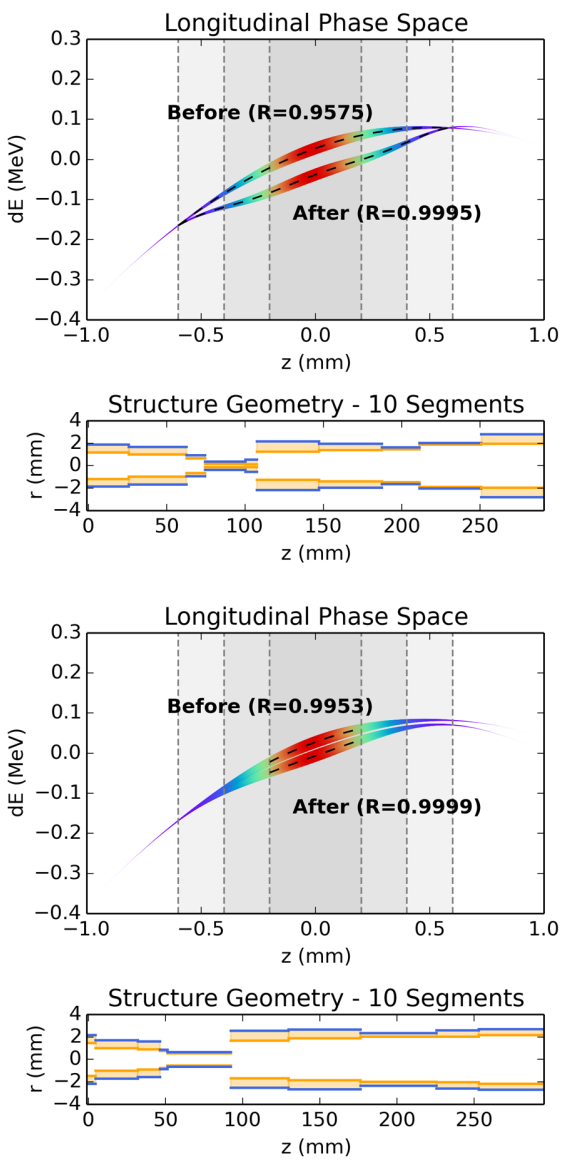
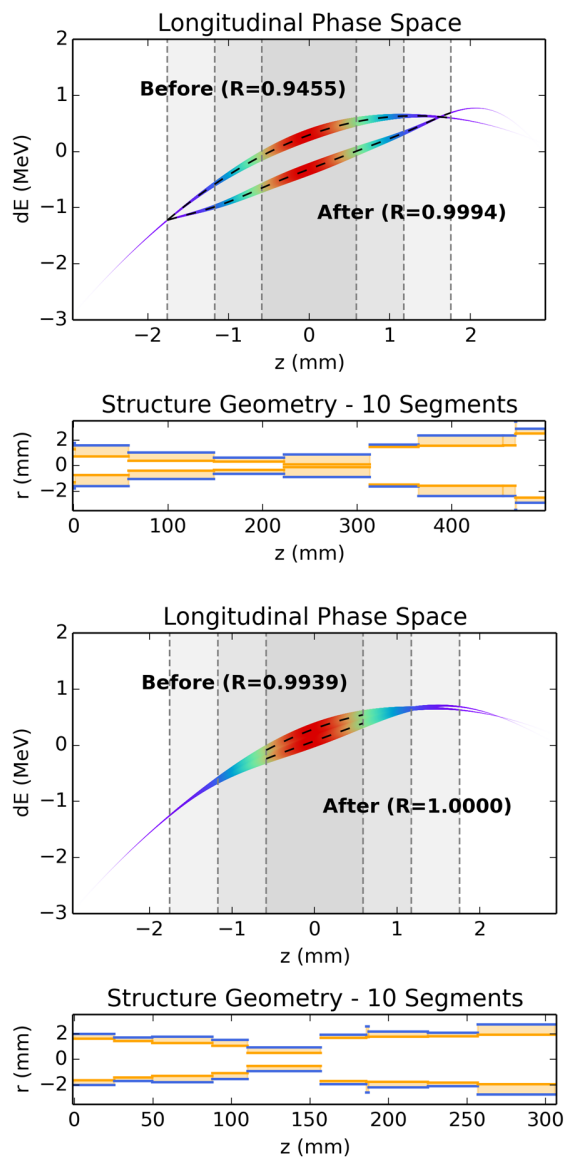
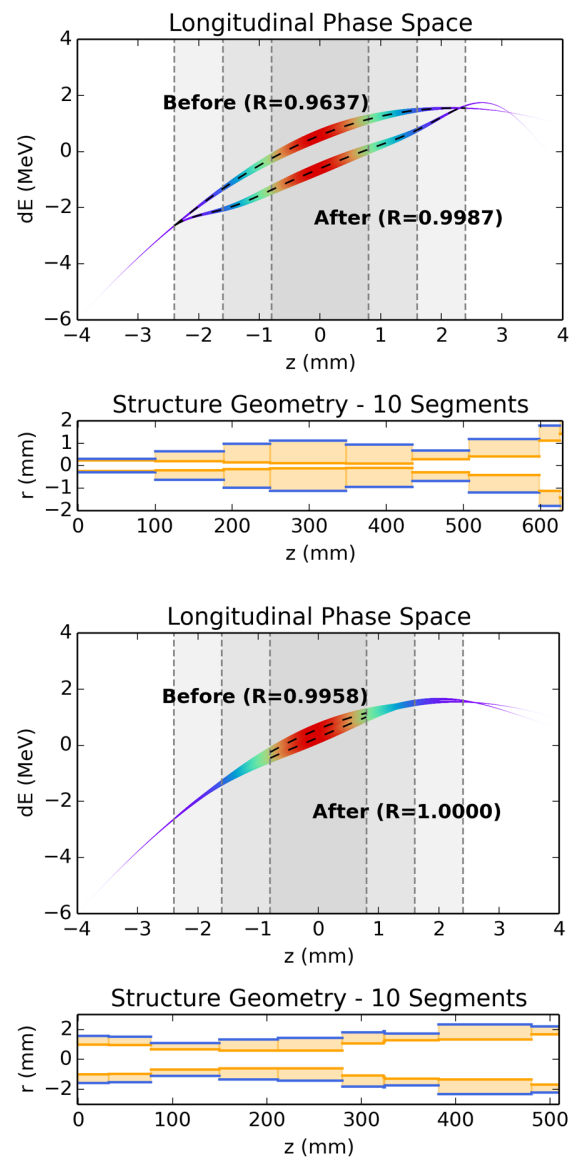

FIG. 8. LPSS optimization results for input LPSs based on the ARES working points shown in Table II. The optimization goal was to achieve $R=1$ across the full $6 \sigma$ ROI (top row), as well as a centered $2 \sigma$ ROI (bottom row). From left to right: WP1, WP2, WP3. Each plot shows the LPS before and after the LPSS interaction. The color and thickness visualize the current profile. The gray shaded areas correspond to the 2, 4 and $6 \sigma$ regions respectively. The head of the bunch is on the left (negative $z$ values). Below the main plot, the geometry of the final segmented DLW is visualized, with the orange line corresponding to the inner radius and the blue line to the outer radius. 
geometries of the resulting LPSS structures are shown. The shorter in time the input LPS, the shorter the resulting LPSS. This is partly due to the smaller required modulation depth, but also due to how the wakefield amplitude scales with the required inner radii of the segments. In order to accommodate a typical focused beam envelope, the individual segments of the LPSS structures are sorted such that the tightest segment is placed at the center of the structure, which then has increasing inner radii towards both entrance and exit. The results show that a similar degree of linearization can be achieved, regardless of the different bunch lengths across the different working points. The shape of the respective resulting structure does vary significantly however, due to the required modal content.

\section{A. Other optimization goals}

As already discussed above, not only the linearization within a defined ROI can be set as an optimization goal. Another interesting case could be the removal of any correlated energy spread, aiming for a completely flat LPS. Figure 9 shows the result of such an optimization, based on the $10 \mathrm{pC}$ WP1 as shown in Table II. It can be seen that the phase space is significantly flattened within the $4 \sigma$ ROI. Note that this kind of structure could be used to prepare an LPS for further modulation as shown, for example, in Sec. III.
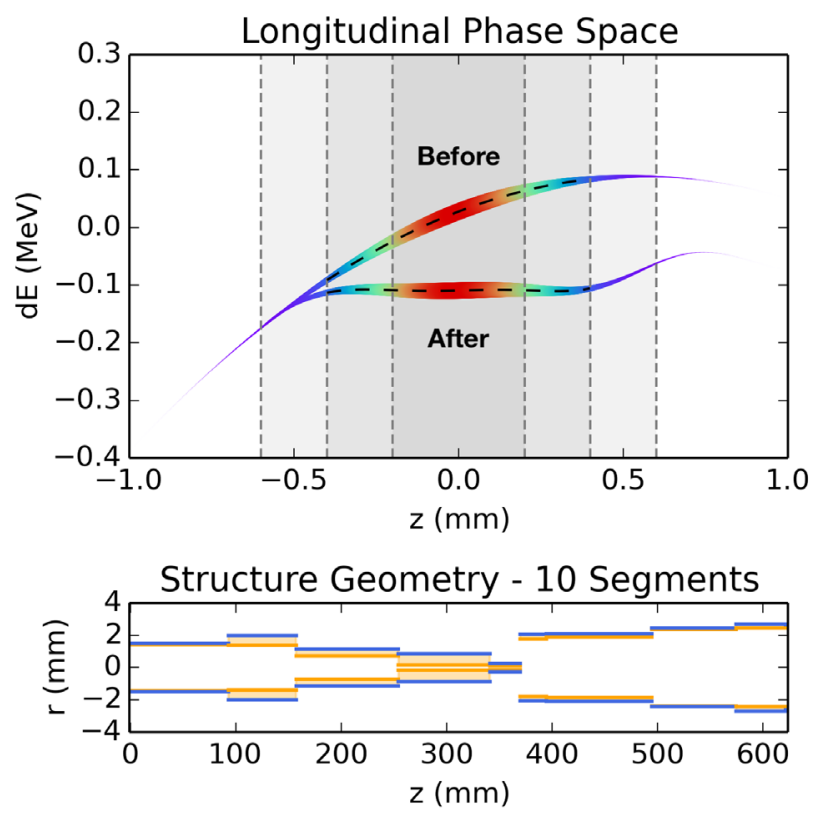

FIG. 9. LPSS optimization results based on the ARES working point WP1 shown in Table II. The optimization goal was to completely remove any correlated energy spread within a $4 \sigma$ ROI. The color and thickness visualize the current profile. The gray shaded areas correspond to the 2, 4 and $6 \sigma$ regions respectively. The head of the bunch is on the left (negative $z$ values). Below the main plot, the geometry of the final segmented DLW is visualized, with the orange line corresponding to the inner radius and the blue line to the outer radius.

\section{B. Example case: Bunch compression}

It was shown in simulation that at ARES, based on magnetic compression and a slit collimator, sub-fs bunch lengths can be achieved [37,38]. Starting from an initial bunch charge of $20 \mathrm{pC}$ a final rms bunch length of $0.51 \mathrm{fs}$ was achieved, $1.75 \mathrm{~m}$ downstream of the chicane exit (cf. Fig. 6). The remaining charge after the slit is $0.79 \mathrm{pC}$, which corresponds to a $\sim 4 \%$ transmission. The full set of beam parameters is summarized in the first column of Table III. Here we aim to show that based on using an LPSS before magnetic bunch compression, we can achieve similar beam parameters, but at higher mean energy and higher final peak current. To this end WP4, which is a modified version of WP1 (cf. Table II), where the TWS structures are driven at $-38^{\circ}$ is used in a start-to-end simulation using ASTRA, the LPSS optimization routine and IMPACT-T [39]. Up to the LPSS structure the simulation includes space charge forces via ASTRA and after that both space charge and coherent synchrotron radiation (CSR) via IMPACT-T. Full linearization in a $4 \sigma$ optimization ROI was considered as the LPSS optimization goal. The resulting beam parameters $1.75 \mathrm{~m}$ downstream of the chicane exit are summarized in Table III, where WP4 refers to our working point without LPSS linearization and WP4, $4 \sigma$ to the case employing the optimized LPSS structure. The final longitudinal phase spaces are shown in Fig. 10. It can be seen that using a passive LPSS structure upstream of the magnetic bunch compressor in $\tilde{n} \sigma$ linearization mode yields bunches with comparable beam quality, but at $26 \%$ higher mean energy. At the same time, even though the initial charge is $50 \%$ less, the final charge is higher, due to the larger slit width. This is enabled by the high degree of linearization in the LPSS ROI. The peak current is noticeably higher in both WP4 cases ( $2 \times$ without the LPSS and $\sim 3.5 \times$ using the LPSS).

TABLE III. Beam parameters of different ARES working points (WP) $1.75 \mathrm{~m}$ downstream of the chicane exit $(z=30.5 \mathrm{~m})$. WP, Zhu taken from [37], WP4 obtained from ASTRA and IMPACT-T simulations (no LPSS interaction). WP4, $4 \sigma$ refers to WP4 + LPSS optimization with a $4 \sigma$ ROI. TWS: traveling wave structure.

\begin{tabular}{lccc}
\hline \hline Parameter & $\mathrm{WP}, \mathrm{Zhu}$ & $\mathrm{WP} 4$ & $\mathrm{WP} 4,4 \sigma$ \\
\hline Initial charge & $20 \mathrm{pC}$ & $10 \mathrm{pC}$ & $10 \mathrm{pC}$ \\
Final charge & $0.79 \mathrm{pC}$ & $2.2 \mathrm{pC}$ & $2.18 \mathrm{pC}$ \\
TWS injection phase & $-53^{\circ}$ & $-38^{\circ}$ & $-38^{\circ}$ \\
Chicane $R_{56}$ & $-12.4 \mathrm{~mm}$ & $-22.2 \mathrm{~mm}$ & $-22.2 \mathrm{~mm}$ \\
Chicane slit width & $0.4 \mathrm{~mm}$ & $0.6 \mathrm{~mm}$ & $0.6 \mathrm{~mm}$ \\
$E_{0}$ & $100.5 \mathrm{MeV}$ & $126.0 \mathrm{MeV}$ & $126.5 \mathrm{MeV}$ \\
$\sigma_{E} / E_{0}$ & $1.7 \times 10^{-3}$ & $2.5 \times 10^{-3}$ & $2.5 \times 10^{-3}$ \\
$\sigma_{t}$ & $0.51 \mathrm{fs}$ & $0.84 \mathrm{fs}$ & $0.73 \mathrm{fs}$ \\
$\varepsilon_{\mathrm{n}, x}$ & $0.11 \mu \mathrm{m}$ & $0.35 \mu \mathrm{m}$ & $0.35 \mu \mathrm{m}$ \\
$\varepsilon_{\mathrm{n}, y}$ & $0.1 \mu \mathrm{m}$ & $0.17 \mu \mathrm{m}$ & $0.13 \mu \mathrm{m}$ \\
$I_{\mathrm{p}}$ & $0.62 \mathrm{kA}$ & $1.32 \mathrm{kA}$ & $2.18 \mathrm{kA}$ \\
\hline \hline
\end{tabular}



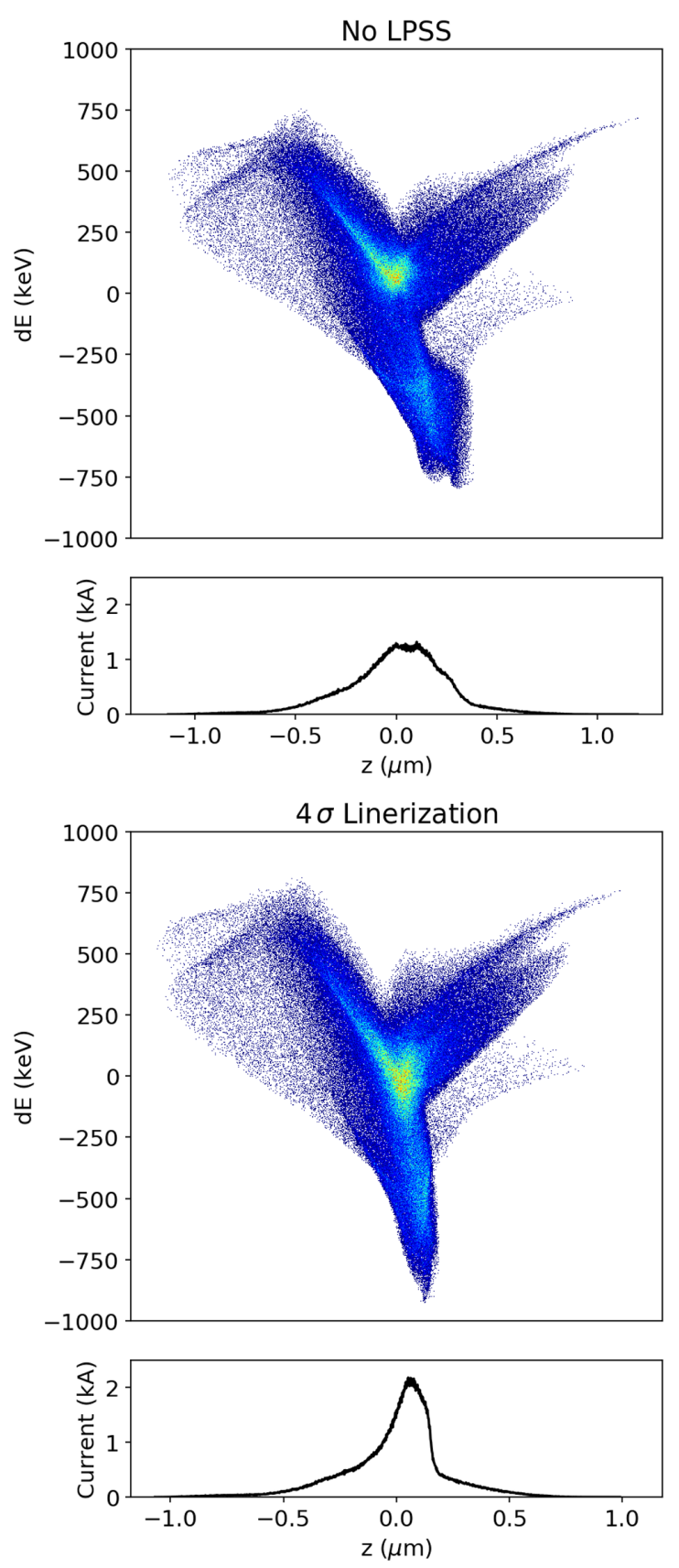

FIG. 10. Numerical simulation of the longitudinal phase space and current profile of the ARES working point WP4 shown in Table III, $1.75 \mathrm{~m}$ downstream of the chicane exit $(z=30.5 \mathrm{~m})$. Top: bunch compression without applying the LPSS optimization, i.e., no structure. Bottom: bunch compression after applying a $4 \sigma$ linearization with an optimized LPSS structure.

We note that the transverse phase space of WP4 was not fully optimized as part of this study, which means that the transverse properties of the beam could be improved in future iterations of this particular working point.

Finally, it should be noted that at higher overall charges significant energy modulation due to CSR can spoil the linearity of the LPS during bunch compression. This, however, could be included into future versions of the LPSS optimization routine as the virtual last element of the LPSS structure, or by considering other mitigation concepts [40].

\section{REALISTIC STRUCTURES}

As mentioned in Sec. II, an LPSS can be either thought of as a cascaded arrangement of multiple DLWs or one single segmented structure. The optimization results discussed above show that the final geometry of the LPSS structure can be complicated, with arbitrary combinations of inner radii and dielectric thicknesses. In this work we have limited the number of segments to $N=10$, but this number can be chosen to be even larger, resulting in a potentially even more complicated structure shape. We therefore propose using 3D printers for manufacturing LPSS structures. This way, the LPSS structure can be printed as one monolithic structure with feature sizes down to tens of micrometers. A monolithic approach is best in terms of transverse alignment of the individual segments. The material can either be vacuum compatible plastic or even quartz [41]. The dielectric structure obtained from the printer can then subsequently be metallized. In addition, 3D printing allows for rapid prototyping.

\section{A. Segment transitions}

Our previous discussion has treated the LPSS as a series of individual successive DLW segments. In order to calculate the resulting energy modulation, the individual wakefields of the segments were summed up and applied to the input LPS. Although this is a good first approximation, in reality there are two issues with this approach. First, the sharp transitions between the segments will disturb the wakefield slightly. Second and most importantly, this kind of segmented structure cannot be produced, because in some cases it turns out that $a_{i+1}>b_{i}$, which would mean that the $(i+1)$ th segment could not actually be attached to the $i$ th segment. It is hence necessary to include transition elements between the individual segments. These elements could for example be short linearly tapered sections. Although adding such a transition would enable production of the structure, it also alters the resulting wakefield. In order to investigate this effect, ECHO2D [42] simulations were performed. The longitudinal monopole wakefield, excited by a Gaussian current with an arbitrarily chosen $\sigma_{t}=500 \mathrm{fs}$, was compared for three different cases:

(1) the sum of the resulting wakefield of two individually simulated DLW segments of length $l_{1}$ and $l_{2}$,

(2) the two segments directly behind one another (sharp, unrealistic transition), and

(3) the two segments connected with a linearly tapered transition region of length $l_{t}$.

Note that the overall length $L$ of the structure is the same for both cases 2 and 3. This means that for case 3 the 

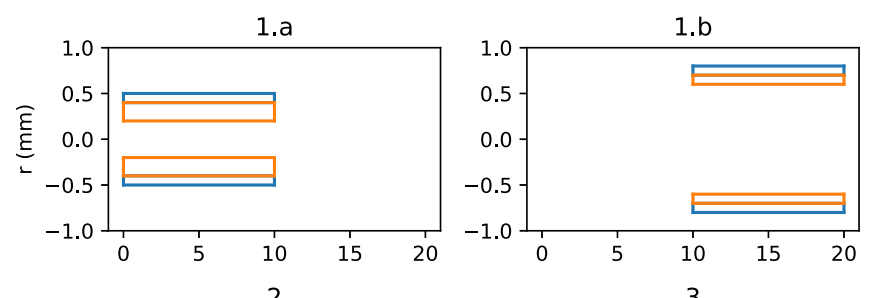

current. It has to be noted that although this minimum does not depend strongly on the longitudinal dimensions of the segments, it does depend on the transverse dimensions $a_{i}$ and $b_{i}$ (and on $\sigma_{t}$, as the whole composition of the structure depends on it). It is hence implied that each transition has to be uniquely optimized. This, however, can be directly factored into the optimization routine discussed above (extending the number of degrees of freedom from $3 N$ to $4 N-1)$.

It was shown that the integrated difference between a case 1 and 3 geometry can be minimized by adjusting $l_{t}$. Figure 13 shows the longitudinal wake for all three geometry cases based on a simulation using the exemplary parameters from above and an optimized $l_{t}$ of $953 \mu \mathrm{m}$. In addition to the wakefields, the absolute and relative differ-

FIG. 11. Illustration of the DLW geometry used in the ECHO2D simulations. All cases include a (lossless) metal coating of $100 \mu \mathrm{m}$ thickness. The blue lines correspond to the outline of the metal coating and the orange lines to the outline of the dielectric. (1.a) Single segment of length $l_{1}=10 \mathrm{~mm}$. (1.b) Single segment of length $l_{2}=10 \mathrm{~mm}$. (2) Segments right next to each other (sharp, unrealistic transition). (3) Two segments connected with a linearly tapered transition region of length $l_{t}=1 \mathrm{~mm}$.

individual segments are shortened by $0.5 \cdot l_{t}$ each. Hence, case 2 is essentially case 3 with $l_{t}=0$. See Fig. 11 for an illustration of the three different cases.

Figure 12 shows the integrated residual difference between the wakefield obtained from the case 1 and case 3 geometries using a drive bunch with $\sigma_{t}=500 \mathrm{fs}$ vs different values of $l_{t}$. The exemplary dimensions of the DLW segments are $a_{1}=0.2 \mathrm{~mm}, b_{1}=0.4 \mathrm{~mm}, l_{1}=10 \mathrm{~mm}$, $a_{2}=0.6 \mathrm{~mm}, b_{2}=0.7 \mathrm{~mm}, l_{2}=10 \mathrm{~mm}$. The dielectric is defined by $\epsilon_{r}=4.41, \mu_{r}=1$ and the metal coating, which is assumed to be a perfect conductor, has a thickness of $0.1 \mathrm{~mm}$. The simulation results show that an optimal $l_{t}$ can be found depending on the area of interest around the peak of the drive

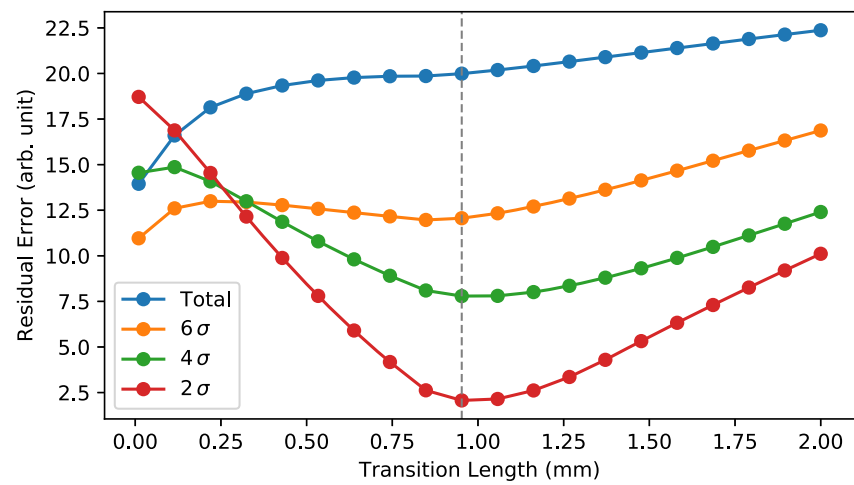

FIG. 12. Integrated residual difference between the wakefield obtained from the sum of two singular DLW segments and a combined device with a linearly tapered transition region of length $l_{t}$, as shown in Fig. 11. The different curves correspond to the $6 \sigma, 4 \sigma$ and $2 \sigma$ parts of the drive bunch, as well as the complete simulation box (total). The data is normalized to the respective ROI size for comparability. ence compared to case 1 is plotted for both the case 2 and case 3 geometry respectively. It can be seen that, depending on the area of interest along the drive bunch, the error can be very small and is generally smaller than $10 \%$. The error can be large, however, towards the tail of the bunch. The significance of this effect depends a lot on the specific input electron distribution and the particular use case. Assuming a Gaussian longitudinal current profile, $<16 \%$ of the charge is affected. Recalling Fig. 12, the goal should in general be to minimize the effect of the transition in the region of highest charge density. In summary, it can be concluded that it is possible to find transition regions, which minimize the difference of the produced wakefield compared to the summed up wakefield of individual segments, as used in the optimization routine discussed above.
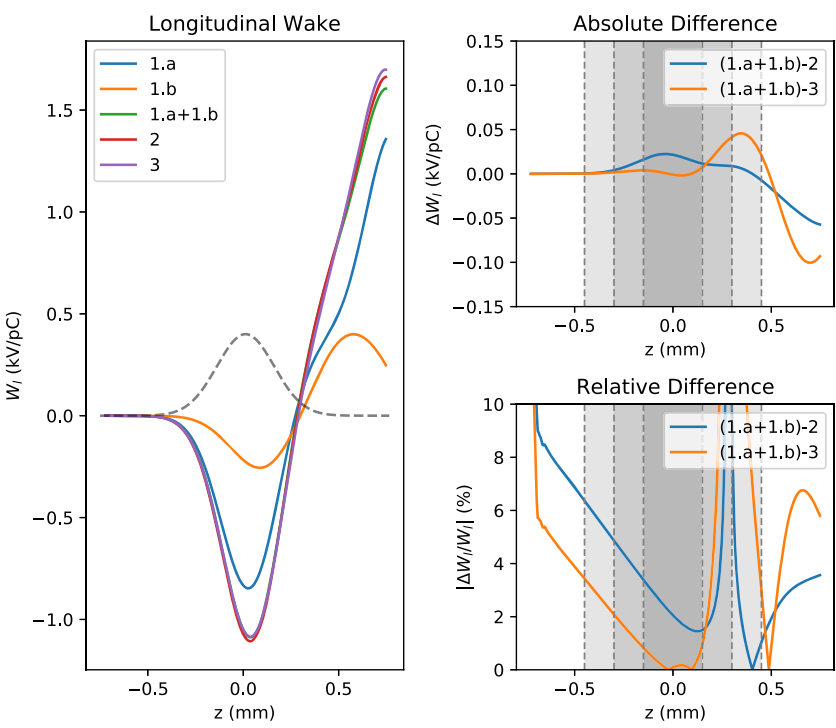

FIG. 13. Comparison of the wakefield obtained using the geometries illustrated in Fig. 11. $l_{t}=953 \mu \mathrm{m}$, which is the value determined by the optimization scan shown in Fig. 12. The shaded areas correspond to the $6 \sigma, 4 \sigma$ and $2 \sigma$ parts of the drive bunch. The legends refer to the notation established in Fig. 11. 


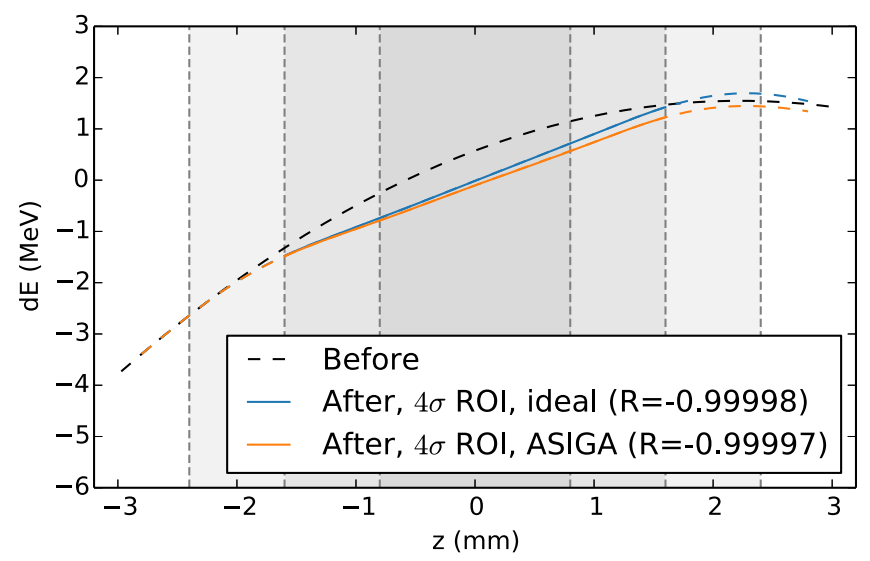

FIG. 14. Comparison of LPSS optimization results for a Gaussian input current profile. The optimization goal was to achieve $R=1$ in a $4 \sigma$ region of interest. The input beam parameters correspond to WP3 (see Table II). Blue: ideal LPSS; orange: LPSS taking a lateral printing resolution of $27 \mu \mathrm{m}$ and a longitudinal printing resolution of $10 \mu \mathrm{m}$ into account (as can, for example, be achieved with an ASIGA MAX X27 3D printer).

\section{B. Manufacturing}

The optimization shown above does not include any assumption about possible inaccuracies due to the manufacturing process. In reality, the exact shape of the individual segments is determined by the tolerances during production. Assuming a 3D-printed structure, the parameters $a_{i}, b_{i}$ and $l_{i}$ are determined by the transverse and longitudinal printing resolution and on how the structure is printed (flat or standing). We consider the ASIGA MAX $\mathrm{X} 27$ 3D printer [43] and its printing resolution as an example. This particular printer has a longitudinal resolution $\rho_{z}$ of $10 \mu \mathrm{m}$ (minimum layer thickness) and a lateral resolution $\rho_{x y}$ of $27 \mu \mathrm{m}$ (Digital Light Processing pixel size). Figure 14 shows the comparison between the linearization using an ideal LPSS and an LPSS, which was optimized taking the aforementioned printing resolution into account. Here we model the effect such that $\tilde{a}_{i}=\left\lfloor 2 a_{i} / \rho_{x y}\right\rfloor \cdot \rho_{x y} / 2, \quad \tilde{b}_{i}=\left\lceil 2 b_{i} / \rho_{x y}\right\rceil \cdot \rho_{x y} / 2 \quad$ and $\tilde{l}_{i}=\left\lceil l_{i} / \rho_{z}\right\rceil \cdot \rho_{z}$, where the tilde denotes the radii and length of the segments after applying the printer resolution. The results show that the limited printing resolution only has a small impact on the final linearization. It has to be noted that the chirp across the ROI is different, but only because it was not part of the particular optimization goal.

\section{ROBUSTNESS OF THE SCHEME}

As discussed above, an LPSS must be specifically tailored to the incoming LPS. In reality the actual shape of the input LPS varies according to the stability of certain accelerator machine parameters. The LPS in particular is influenced by the stability of both amplitude and phase of the accelerating fields, but also by dispersive sections and collective effects, such as CSR. It is hence interesting to investigate the effect of the actual shape of the input LPS on the output LPS. To this end, the numerically simulated third ARES working point (WP3) with $200 \mathrm{pC}$ of total charge and a Gaussian time profile with $\sigma_{t}=2.65 \mathrm{ps}$ (see Sec. IV) is used as the LPSS input. For a fixed LPSS geometry, the sensitivity of the linearity parameter $R$ within a $4 \sigma$ ROI is determined for four different parameters, with the first two parameters being the amplitude and phase of the accelerating field, which define the curvature of the incoming LPS. The third parameter is $\sigma_{t}$, which in reality, of course, nontrivially depends on multiple factors, but is here varied independently, while keeping the total bunch charge constant. The fourth parameter is the bunch charge $Q$, keeping $\sigma_{t}$ constant. The top four plots in Fig. 15 summarize the results of the respective scans. The results show that the relative change in $R$ is very little ( $\ll 0.1 \%)$, leading to the conclusion that, in the specific case of the example of LPS linearization, the LPSS scheme is robust within the limits of typical accelerator machine stability.

In addition to the uncertainty in the shape of the input LPS, there can also be systematic errors in the geometry of the LPSS itself. In order to investigate this, two scenarios were studied. The first one is a constant error $\Delta r$ of both the inner and outer radii, i.e., $\tilde{a}_{i}=a_{i}+\Delta r$ and $\tilde{b}_{i}=b_{i}+\Delta r$.
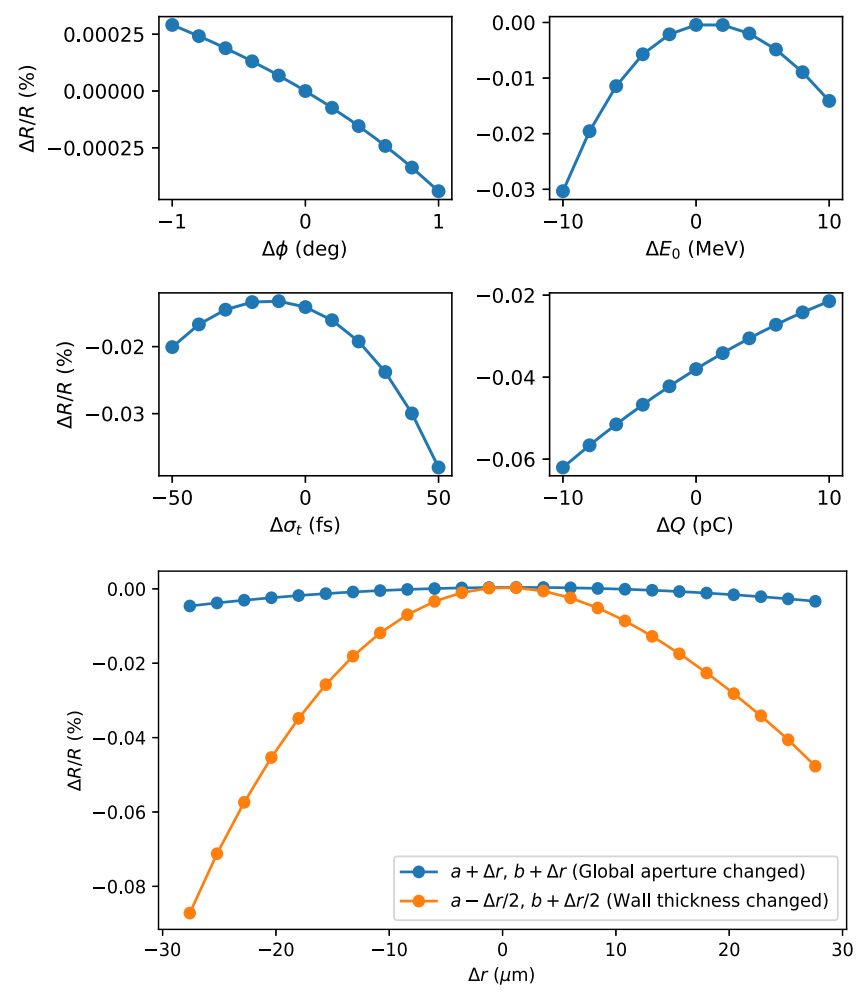

FIG. 15. Top four plots: relative change of the linearity factor $R$ vs four different parameters, which influence the input LPS. Bottom plot: relative change of the linearity factor $R$ vs a constant error $\Delta r$ for two different systematic manufacturing error scenarios. The same $\Delta r$ is applied to all segments. 
The second scenario is a constant difference in wall thickness, meaning $\tilde{a}_{i}=a_{i}-\Delta r / 2$ and $\tilde{b}_{i}=b_{i}+\Delta r / 2$. The range is chosen to be according to the lateral resolution of the ASIGA printer discussed above. Hence $\Delta r \in[-30,30] \mu \mathrm{m}$. The bottom plot in Fig. 15 summarizes the results of the scan. The LPSS optimization scenario is the same as before (WP3, $4 \sigma$ ROI). It can be seen that the change in global aperture has a very small effect on $R$ $(<0.01 \%)$. The wall thickness, on the other hand, has a $\sim 10 \times$ stronger effect, with a slight asymmetry. It is still a small effect with $|\Delta R|<0.1 \%$ within the given range of $\Delta r$. The slightly asymmetric behavior might be explained by the nonlinear dependence of the amplitude and frequency of the wake towards smaller inner radii (cf. Fig. 2) in conjunction with an increase in the modal content as the thickness of the dielectric lining increases. A more thorough study of this behavior would be interesting, but exceeds the scope of this work.

\section{CONCLUSION AND OUTLOOK}

A completely passive LPSS solution, based on segmented DLWs, was presented and studied both analytically and numerically. The results based on the idealized single-mode Fourier synthesis, coupled with a longitudinally dispersive section, reveal phase space configurations, which could be interesting for applications, especially in the context of radiation generation (multicolor microbunch trains, submicrobunches with tunable relative spacing, etc.).

Arbitrary multimode optimization was investigated, which enables application of the method to arbitrary input phase spaces. The results shown here are promising, as the exemplary goal of full linearization of the input LPS within a given $\tilde{n} \sigma_{z}$ ROI of an input LPS was achieved in a semianalytical simulation to a very high degree. The input LPSs used for the study were chosen to be realistic and are based on numerical simulation of an existing accelerator, the ARES linac at DESY. Motivated by these results, a start-to-end simulation of a possible experiment at the ARES linac was performed yielding sub-fs bunches comparable to reference working points, but at $\sim 26 \%$ higher mean energy and $\sim 3.5 \times$ larger peak current, starting from $50 \%$ less initial charge.

It was furthermore shown, based on ECHO2D simulations, that it is possible to integrate short transition regions between the segments, which enables realistic structure shapes that can be produced with a 3D printer. The optimization routine used in this work can export its result as 3D models suitable for direct import into a 3D printing software. The structures can be made from metallized 3Dprinted plastic, or even 3D-printed quartz [41]. Depending on the specific printing process, longer structures might be constructed of two or more cascaded macro segments.

The robustness of the scheme was investigated for the LPS linearization example and found to be satisfactory based on accelerator stability, as well as manufacturing tolerance considerations. This together with the low cost of the devices alleviates the fact that each LPSS device is specific to a given accelerator working point; multiple structures could be installed and swapped in as needed.

Further studies could focus on transverse effects in LPSS structures, as potentially triggered dipole modes might lead to deflection. Also, material-dependent charging of the dielectric could be studied. Finally, the LPSS optimization routine could be updated to take expected downstream LPS modulation, due to e.g., collective effects, into account.

\section{ACKNOWLEDGMENTS}

The authors would like to thank I. Zagorodnov for support in the use of ECHO2D.

[1] R. J. England, J. B. Rosenzweig, and G. Travish, Generation and Measurement of Relativistic Electron Bunches Characterized by a Linearly Ramped Current Profile, Phys. Rev. Lett. 100, 214802 (2008).

[2] P. Piot, C. Behrens, C. Gerth, M. Dohlus, F. Lemery, D. Mihalcea, P. Stoltz, and M. Vogt, Generation and Characterization of Electron Bunches with Ramped Current Profiles in a Dual-Frequency Superconducting Linear Accelerator, Phys. Rev. Lett. 108, 034801 (2012).

[3] F. Lemery and P. Piot, Tailored electron bunches with smooth current profiles for enhanced transformer ratios in beam-driven acceleration, Phys. Rev. ST Accel. Beams 18, 081301 (2015).

[4] G. Andonian, S. Barber, F. H. O'Shea, M. Fedurin, K. Kusche, C. Swinson, and J. B. Rosenzweig, Generation of Ramped Current Profiles in Relativistic Electron Beams Using Wakefields in Dielectric Structures, Phys. Rev. Lett. 118, 054802 (2017).

[5] P. Muggli, V. Yakimenko, M. Babzien, E. Kallos, and K. P. Kusche, Generation of Trains of Electron Microbunches with Adjustable Subpicosecond Spacing, Phys. Rev. Lett. 101, 054801 (2008).

[6] P. Piot, Y.-E. Sun, J. G. Power, and M. Rihaoui, Generation of relativistic electron bunches with arbitrary current distribution via transverse-to-longitudinal phase space exchange, Phys. Rev. ST Accel. Beams 14, 022801 (2011).

[7] G. Ha, M. H. Cho, W. Namkung, J. G. Power, D. S. Doran, E. E. Wisniewski, M. Conde, W. Gai, W. Liu, C. Whiteford, Q. Gao, K.-J. Kim, A. Zholents, Y.-E. Sun, C. Jing, and P. Piot, Precision Control of the Electron Longitudinal Bunch Shape Using an Emittance-Exchange Beam Line, Phys. Rev. Lett. 118, 104801 (2017).

[8] G. Loisch, J. Good, M. Gross, H. Huck, I. Isaev, M. Krasilnikov, O. Lishilin, A. Oppelt, Y. Renier, F. Stephan, R. Brinkmann, F. Grüner, and I. Will, Photocathode laser based bunch shaping for high transformer ratio plasma wakefield acceleration, Nucl. Instrum. Methods Phys. Res., Sect. A 909, 107 (2018); 3rd European Advanced Accelerator Concepts Workshop, EAAC2017.

[9] P. Craievich, Passive longitudinal phase space linearizer, Phys. Rev. ST Accel. Beams 13, 034401 (2010). 
[10] P. Emma, M. Venturini, K. L. F. Bane, G. Stupakov, H.-S. Kang, M. S. Chae, J. Hong, C.-K. Min, H. Yang, T. Ha, W. W. Lee, C. D. Park, S. J. Park, and I. S. Ko, Experimental Demonstration of Energy-Chirp Control in Relativistic Electron Bunches Using a Corrugated Pipe, Phys. Rev. Lett. 112, 034801 (2014).

[11] G. Penco, E. Allaria, I. Cudin, S. Di Mitri, D. Gauthier, S. Spampinati, M. Trovó, L. Giannessi, E. Roussel, S. Bettoni, P. Craievich, and E. Ferrari, Passive Linearization of the Magnetic Bunch Compression Using Self-Induced Fields, Phys. Rev. Lett. 119, 184802 (2017).

[12] S. Antipov, M. Babzien, C. Jing, M. Fedurin, W. Gai, A. Kanareykin, K. Kusche, V. Yakimenko, and A. Zholents, Subpicosecond Bunch Train Production for a Tunable mJ Level THz Source, Phys. Rev. Lett. 111, 134802 (2013).

[13] F. Lemery, P. Piot, G. Amatuni, P. Boonpornprasert, Y. Chen, J. Good, B. Grigoryan, M. Groß, M. Krasilinikov, O. Lishilin, G. Loisch, A. Oppelt, S. Philipp, H. Qian, Y. Renier, F. Stephan, and I. Zagorodnov, Passive Ballistic Microbunching of Nonultrarelativistic Electron Bunches Using Electromagnetic Wakefields in Dielectric-Lined Waveguides, Phys. Rev. Lett. 122, 044801 (2019).

[14] L. H. Yu, Generation of intense uv radiation by subharmonically seeded single-pass free-electron lasers, Phys. Rev. A 44, 5178 (1991).

[15] G. Stupakov, Using the Beam-Echo Effect for Generation of Short-Wavelength Radiation, Phys. Rev. Lett. 102, 074801 (2009).

[16] D. Xiang and G. Stupakov, Echo-enabled harmonic generation free electron laser, Phys. Rev. ST Accel. Beams 12, 030702 (2009).

[17] D. Xiang, E. Colby, M. Dunning, S. Gilevich, C. Hast, K. Jobe, D. McCormick, J. Nelson, T. O. Raubenheimer, K. Soong, G. Stupakov, Z. Szalata, D. Walz, S. Weathersby, M. Woodley, and P.-L. Pernet, Demonstration of the EchoEnabled Harmonic Generation Technique for Short-Wavelength Seeded Free Electron Lasers, Phys. Rev. Lett. 105, 114801 (2010).

[18] D. Xiang, E. Colby, M. Dunning, S. Gilevich, C. Hast, K. Jobe, D. McCormick, J. Nelson, T. O. Raubenheimer, K. Soong, G. Stupakov, Z. Szalata, D. Walz, S. Weathersby, and M. Woodley, Evidence of High Harmonics from EchoEnabled Harmonic Generation for Seeding X-Ray Free Electron Lasers, Phys. Rev. Lett. 108, 024802 (2012).

[19] N. Sudar, P. Musumeci, I. Gadjev, Y. Sakai, S. Fabbri, M. Polyanskiy, I. Pogorelsky, M. Fedurin, C. Swinson, K. Kusche, M. Babzien, and M. Palmer, Demonstration of Cascaded Modulator-Chicane Microbunching of a Relativistic Electron Beam, Phys. Rev. Lett. 120, 114802 (2018).

[20] E. Hemsing and D. Xiang, Cascaded modulator-chicane modules for optical manipulation of relativistic electron beams, Phys. Rev. ST Accel. Beams 16, 010706 (2013).

[21] L. Zhao, T. Jiang, C. Lu, R. Wang, Z. Wang, P. Zhu, Y. Shi, W. Song, X. Zhu, C. Jing, S. Antipov, D. Xiang, and J. Zhang, Few-femtosecond electron beam with terahertzfrequency wakefield-driven compression, Phys. Rev. Accel. Beams 21, 082801 (2018).

[22] G. Voss and T. Weiland, The wakefield acceleration mechanism, DESY Report No. DESY 82-074, 1982.
[23] M. Rosing and W. Gai, Longitudinal- and transverse-wakefield effects in dielectric structures, Phys. Rev. D 42, 1829 (1990).

[24] K.-Y. Ng, Wake fields in a dielectric-lined waveguide, Phys. Rev. D 42, 1819 (1990).

[25] M. I. Ivanyan, L. V. Aslyan, K. Floettmann, F. Lemery, and V. M. Tsakanov, Wakefields in conducting waveguides with a lossy dielectric channel, Phys. Rev. Accel. Beams 23, 041301 (2020).

[26] V. Paramonov and K. Floettmann, Lower limit of the transverse emittance growth in deflecting rf fields, Phys. Rev. Accel. Beams 23, 014401 (2020).

[27] A. Chao, Physics of collective instabilities in high-energy accelerators, Physics of Collective Instabilities in HighEnergy Accelerators, Wiley Series in Beams and Accelerator Technologies (John Wiley and Sons, New York, 1993).

[28] G. V. Stupakov, Wake and impedance, Report No. SLACPUB-8683, 2000 (unpublished), available from the Stanford Linear Accelerator Center.

[29] P. Piot, J. Wang, and F. Lemery, Wakefield in axisymmetric dielectric-lined waveguides, PYTHON program available from GitHub at https://github.com/NIUaard/DiWakeCyl.

[30] I. Will and G. Klemz, Generation of flat-top picosecond pulses by coherent pulse stacking in a multicrystal birefringent filter, Opt. Express 16, 14922 (2008).

[31] F. Lemery and P. Piot, Ballistic bunching of photoinjected electron bunches with dielectric-lined waveguides, Phys. Rev. ST Accel. Beams 17, 112804 (2014).

[32] E. Hemsing, G. Stupakov, D. Xiang, and A. Zholents, Beam by design: Laser manipulation of electrons in modern accelerators, Rev. Mod. Phys. 86, 897 (2014).

[33] R. Eberhart and J. Kennedy, A new optimizer using particle swarm theory, in MHS'95. Proceedings of the Sixth International Symposium on Micro Machine and Human Science, Nagoya, Japan (IEEE, 1995), pp. 39-43, https:// doi.org/10.1109/MHS.1995.494215.

[34] http://www.pyopt.org, accessed 2019.

[35] K. Floettmann, ASTRA-A space charge tracking algorithm, http://www.desy.de/ mpyflo.

[36] B. Marchetti, R. Assmann, U. Dorda, F. Burkart, E. Panofski, P. A. Walker, F. Lemery, D. Marx, F. Mayet, M. Kellermeier, S. Yamin, S. Jaster-Merz, W. Kuropka, F. Jafarinia, K. Floettmann, R. Brinkmann, and W. Hillert, SINBAD-ARES - A Photo-Injector for external Injection Experiments in novel Accelerators at DESY, J. Phys.: Conf. Ser. 1596, 012036 (2020).

[37] J. Zhu, Design study for generating subfemtosecond to femtosecond electron bunches for advanced accelerator development at SINBAD, Ph.D. thesis, University of Hamburg, Hamburg, Germany, 2017).

[38] J. Zhu, R. W. Assmann, M. Dohlus, U. Dorda, and B. Marchetti, Sub-fs electron bunch generation with sub-10-fs bunch arrival-time jitter via bunch slicing in a magnetic chicane, Phys. Rev. Accel. Beams 19, 054401 (2016).

[39] J. Qiang, S. Lidia, R. D. Ryne, and C. Limborg-Deprey, Three-dimensional quasistatic model for high brightness beam dynamics simulation, Phys. Rev. ST Accel. Beams 9, 044204 (2006). 
[40] C. Mitchell, J. Qiang, and P. Emma, Longitudinal pulse shaping for the suppression of coherent synchrotron radiation-induced emittance growth, Phys. Rev. ST Accel. Beams 16, 060703 (2013).

[41] F. Kotz, K. Arnold, W. Bauer, D. Schild, N. Keller, K. Sachsenheimer, T. M. Nargang, C. Richter, D. Helmer, and
B.E. Rapp, Three-dimensional printing of transparent fused silica glass, Nature (London) 544, 337 (2017).

[42] I. Zagorodnov, ECHO2D-Version 3.3, https://echo4d.de/ download/echo2d/.

[43] ASIGA, Max X27, https://www.asiga.com/products/ printers/max_series/max_x/, accessed 2020. 